\title{
Theoretical approach of possibilities of the photothermal thermography using array detector system under random excitation and parametric analysis concerning thermal system identification
}

\author{
S. Oblin, F.X. Wagner et J.L. Bodnar \\ Laboratoire d'Energétique et d'Optique, UFR Sciences Exactes et Naturelles, BP 1039, 51687 Reims cedex 02, \\ France, E-Mail : jl.bodnar@univ-reims.fr
}

\begin{abstract}
The object of the work that we present here, is to approach theoretically the possibilities of thermal system identification, given by the association of a parametric analysis to the photothermal thermography using array detector system.

We show that the method allows the access to a good approximation of pulse and multifrequencies harmonic responses of a duralumin sample as well as to a good identification of its thermophysical properties, while submitting it to a lesser excitation density.
\end{abstract}

\section{Introduction}

The photothermal thermography is a non destructive and contactless method of control, operating at a distance of the analyzed sample. Its principle consists of submitting the sample to be analyzed to a luminous flow, the absorption of which leads to a local rise of temperature to the vicinity of the luminous impact, then to observe emittance variations of the material with the help of an infrared thermography camera. The photothermal signal thus obtained depends on optical and thermophysical properties of the analyzed material, what allows then to characterize them.

Traditionally, this method of non destructive testing is make use under pulse excitation and continuous detection (flash method). It allows, for example, the detection and the dimensional characterization of internal flaw or also the measure of the thermal diffusivity parameter. It presents nevertheless the obligation, to obtain a excitation close to a Dirac function, to use an important energy in a very short time, which does not authorize, for example, the delicate material analysis (biological materials). To allow the study of this last type of materials and therefore to extend the investigation field of the photothermal thermography, new studies and new modes of analysis are still to develop.

Since some years, thanks to development of the computer science and of the methods of signal processing, appear in electronic domain, new methods of system identification, aiming at being complementary to those traditionally used in non destructive testing (Fourier analysis and flash analysis).

Among all these new analysis methods, one seems us particularly interesting for the thermophysical analysis of materials by photothermal thermography, since being able to give access to the pulse response and even to the multifrequencies harmonic response of materials, while submitting them only to a lesser excitation density. It is the parametric analysis.

As the new infrared thermography cameras using array detector systems allow now, the necessary (for this new mode of analysis) continuous acquisition of the photothermal signal, it has seemed us interesting to approach, first theoretical manner, possibilities of the photothermal thermography using array detector, associated to a parametric analysis, concerning measure of thermophysical properties of materials. It is this study that we present here.

We present first the photothermal model developed for the study.

We show then that the method gives access to a good approximation of the pulse and multifrequencies harmonic responses of the studied sample, while submitting it to a lesser excitation density. 
We show finally that this type of analysis allows to determine with a good approximation the different thermophysical properties of a duralumin sample.

\section{The photothermal model developed for the study}

\subsection{The excitation sequence used}

The excitation sequence to be used in our study, had to allow us to extract enough informations from the thermal system studied to build a parametric behavior model as complete as possible.

In order that, it was necessary to choose a excitation type, of course the less disturbing possible, but allowing too, to excite all frequencies contained in the frequency band (of unknown limits) of the thermal system to identify.

Among all possible excitation types, it has seemed us that only a sequence approaching a theoretical white noise, with a perfect frequency spectrum (therefore usable for all types of materials) and being able to remain easily weakly disturbing, would reply to our wish.

Several real excitation sequences approaching this theoretical signal could be used. It is possible to cite the Gauss sequences, the sweep sequences and the pseudo random binary sequences. Essentially for simplicity reasons of utilization, this are these last that we have chosen to use.

The construction of these excitation sequences has unfolded according to the method proposed by J.Auvray [3]. This last consists in collect the researched excitation sequence, to the output of a register composed by D flip flops, curled up via a modulo 2 addition; exits of D flip flops take into account in this addition being those defined by the primitive polynomial associate to the creation procedure (figure 1).

This mode of construction shows that the excitation sequence will be particularly longer and therefore richer in frequencies that the number of $\mathrm{D}$ flip flops $\mathrm{D}$ taken in account will be important. Nevertheless long excitation sequences, perhaps more interesting because allowing to excite more frequencies, lead also to longer calculation time.

In our study, a compromises between sequence length and computation time was therefore to find. It is a number of $10 \mathrm{D}$ flip flops that has seemed us to offer acceptable simulation times, while keeping a sufficiently rich spectrum in frequencies. The length of the used excitation sequence is then 1024 terms and the primitive polynomial taken in account is $x^{10}+x^{3}+1$ (figure 2).

\subsection{The thermal model developed for the study}

The thermal model developed for the study takes, of course, into account the conditions on limits of the photothermal thermography, but also, when it was possible, hypothesis inducing to a formalism and calculations times respectively the simplest and the shortest possible.

Thus, for all of our simulations, we have considered a 1D geometry. We have considered that the sample, with a thickness $\mathrm{L}$, was perfectly insulated on the thermal point of view and that it was initially to the temperature of $0{ }^{\circ} \mathrm{c}$. We have considered that the pseudo - random binary excitation was taken into account by a surface source $g(L, t)$. Finally, we have considered that the temperature measure was made on the side of the excitation (in $\mathrm{x}=\mathrm{L}$ ). These considerations have induced us to the differential system following:

$$
\left\{\begin{array}{lll}
\frac{\partial^{2} T}{\partial x^{2}}-\frac{1}{a} \frac{\partial T}{\partial t}=-\frac{g(L, t)}{k} & 0<\mathrm{x}<\mathrm{L} & \mathrm{t}>0 \\
\frac{\partial T}{\partial x}=0 & \mathrm{x}=0 & \mathrm{t}>0 \\
\frac{\partial T}{\partial x}=0 & \mathrm{x}=\mathrm{L} & \mathrm{t}>0 \\
T=F(x)=0 & 0<\mathrm{x}<\mathrm{L} & \mathrm{t}=0
\end{array}\right.
$$

The complex form of the excitation has leaded us to forsake analytic resolution modes of the heat equation and to consider the analytico - numerical modes of resolution. Among these last, we have chosen to use the Green functions, because generally usable in the case of simple geometry and 
therefore in our case [4]. We have then considered the homogeneous differential system associate to the precedent, that we have solved by the method of variable separation :

$$
\Phi(x, t)=\frac{1}{L} \int_{x^{\prime}=0}^{l}\left[1+2 \sum_{m=1}^{\infty} e^{-a\left(\frac{m \pi}{L}\right)^{2} t} \cos \left(\frac{m \pi x}{L}\right) \cos \left(\frac{m \pi x^{\prime}}{L}\right)\right] F\left(x^{\prime}\right) d x^{\prime}
$$

We have then deduced from this solution the necessary Green function for the resolution of the problem :

$$
G\left(x, t / x^{\prime}, \tau\right)=\frac{1}{L}\left(1+2 \sum_{m=1}^{\infty} e^{-a\left(\frac{m \pi}{L}\right)^{2}(t-\tau)} \cos \left(\frac{m \pi}{L} x\right) \cos \left(\frac{m \pi}{L} x^{\prime}\right)\right)
$$

then the temperature expression required :

$$
T(L, t)=\frac{a}{k L} \int_{\tau=0}^{t}\left(1+2 \sum_{m=1}^{\infty} e^{-a\left(\frac{m \pi}{L}\right)^{2}(t-\tau)}\right) g(\tau) d \tau
$$

\section{The identification mode used for the study}

The system identification mode chosen for our study is from parametric type. The latter consists in construct theoretically the pulse and multifrequencies harmonic responses of the studied physical system, from a parametric behavior model of this last, developed in our case, from the thermal response of the studied sample and from the pseudo - random binary sequence used.

Several types of behavior parametric model could here too be used. As we had no knowledge on the model type to be used to identify our thermal systems, we had to use the most general possible.

We have chosen to make use of an autoregressive average adjusted model (ARMA model, [1]). This type of model considers indeed, that the output signal of the electronic filter, to which is assimilated the thermal system to identify, is linked linear manner to the different states of the input signal of the filter, to the different specific parameters of the filter, and also to the different earlier states of the output signal himself (formula 5). It is therefore very complete, what explains its used in our study.

$$
\widehat{s}(n)=\sum_{m=1}^{p} a_{m} \widehat{s}(n-m)+\sum_{m=0}^{q} b_{m} e(n-m)
$$

The number of parameters (values of $\mathrm{p}$ and $\mathrm{q}$ ) to take in account in our study had to allow us to preserve a good arrangement between precise simulations and calculation time. A number of 30 parameters in input $(\mathrm{p}=30)$ and 30 parameters in outup $(\mathrm{q}=30)$ has seemed to answer to our wish.

Finally, we had to choose the method to be used to identify the different input $\left(a_{n}\right)$ and output $\left(b_{n}\right)$ parameters of the behavior model. For rapidity simplicity of used reasons, it is the lesser square recursive method, that we have chosen.

\section{The theoretical results obtained}

To this stage of the study, we had to our disposition tools allowing to simulate the study of a thermal system by photothermal thermography under random excitation and parametric analysis. We have then tried to approach theoretically possibilities of identification, under lesser energetic constraint, given by this new mode of analysis.

We have first wanted to approach possibilities of the method concerning reconstruction of pulse and multifrequencies photothermal responses (these responses being those classically used to identify thermal systems by photothermal thermography). In order that, we have simulated the random photothermal analysis of a sample of duralumin. We have taken into account the next thermophysical and experimental properties : a thermal conductivity of $169 \mathrm{~W} / \mathrm{mK}$, a density of $2790 \mathrm{~kg} / \mathrm{m}^{3}$, a specific 
heat of $881 \mathrm{~J} / \mathrm{kgK}$, a duralumin thickness of $1 \mathrm{~cm}$, an excitation amplitude of $500 \mathrm{~W} / \mathrm{m}^{2}$ and finally a sampling period of $10 \mathrm{~ms}$ (figure 2).

We have then calculated, in these conditions, the thermal response of the duralumin sample. The obtained result is presented on the figure 3 .

We have then built the behavior model of the sample studied from this thermal response and from the pseudo random binary exciting sequence.

Finally, we have reconstructed the pulse and multifrequencies harmonic response of the sample studied. In order that, in the first case, we have calculated the response of the behavior model for a input signal equal to a theoretical Dirac function $\left(\mathrm{Q}=100 \mathrm{~kJ} / \mathrm{m}^{2}\right)$. In the second case, we have used the Z-transform of the behavior model of behavior (formula 6).

$$
H(j \omega T)=\frac{b_{0}+b_{1}(j \omega T)^{-1}+\ldots+b_{n} \exp (j \omega T)^{-n}}{1-a_{1}(j \omega T)^{-1}-\ldots-a_{n}(j \omega T)^{-n}}
$$

The obtained results are presented on figures 4 and 5 . On the figure 4 , we have compared the pulse response reconstructed by parametric analysis to that calculated with the Parker model of the flash method [5]. The two obtained curves are practically identical. That shows that the parametric method allows well the access, under lesser energetic constraints, to the photothermal pulse response of a thermal system. In addition, this results validate a posteriori the different choice of thermal and parametric model, identification mode and of excitation sequence.

On the figure 5, we have compared the harmonic multifrequencies responses reconstructed by parametric analysis and calculated with the help of the Cowan model [6]. This figure shows close curves of each other without nevertheless being perfectly identical. That shows first, that the parametric method, allows a good approximation of the multifrequencies harmonic response of the duralumin sample studied. That shows also, that a finer choice of analysis conditions (length of exciting sequence, sampling frequency, ...) will have to be researched to allow a thermophysical identification of thermal systems with this type of response.

These first results being encouraging, we have continued our study by verifying that the pulse response reconstructed by parametric analysis allowed well, as the Parker pulse response, to identify the thermophysical properties of the studied sample.

We have first used the method of slope rupture to determine the thermal diffusivity parameter (figure 6). We find a time of slope rupture of approximately $0,46 \mathrm{~s}$, what lead to, according to the Parker formula (formula $7[5,6]$ ), a value of thermal diffusivity of about $69.210^{-6} \mathrm{~m}^{2} / \mathrm{s}$. The real value of this parameter being $68.810^{-6} \mathrm{~m}^{2} / \mathrm{s}$, this result shows the possibilities of the parametric method in matter of thermal diffusivity identification.

$$
\mathrm{a}=\mathrm{L}^{2} / \pi \mathrm{t}_{\mathrm{rp}}
$$

In a second stage, as we knew perfectly (since that imposed theoretically) the value of the density of energy Q deposited to reconstruct the pulse response, we have used the Parker formula giving the expression of the long times asymptotic temperature (formula 8, [5.6]) to determine the value of the density by the specific heat product. We have found a value of approximately $2.4410^{6} \mathrm{~J} / \mathrm{m}^{3} \mathrm{~K}$ (the asymptotic temperature being approximately of $4,1^{\circ} \mathrm{c}$ ), for a real value of $2.4610^{6} \mathrm{~J} / \mathrm{m}^{3} \mathrm{~K}$, what shows that the parametric method, in this particular case, allows also to determine this product of parameters.

$$
\mathrm{T}_{\text {asymptotique }}=\mathrm{Q} / \mathrm{\rho cL}
$$

Finally, we have combined preceding results to end to the identification of the thermal conductivity. We have found a value of $168,8 \mathrm{~W} / \mathrm{mK}$ for a real value of $169 \square \mathrm{W} / \mathrm{mK}$, what also is very acceptable.

\section{Conclusion}


In this work, we have approached theoretically, the possibilities of thermal system identification, at a distance, without contact and under lesser energetic constraint, given by the association of a random excitation and a parametric analysis to the photothermal thermography using array detector system.

We have presented first the photothermal model developed for the study.

We have then shown that the method gives access to a good approximation of the pulse and multifrequencies harmonic responses of the studied sample, while submitting it to a lesser excitation density.

We have finally shown that this type of analysis allows to determine with a good approximation the different thermophysical properties of a duralumin sample.

These results, obtained with a particular sample, are encouraging since appearing open the way to the photothermal identification of delicate materials. They ask now to be generalized and validated experimentally. Studies going in this way are in progress.

\section{References}

[1] J. Max, Traitement du signal. Vol 1 and 2, 1993.

[2] S. Kay, S. Marple, Spectrum analysis :A modern perspective. IEEE, 69, (11), 1991, 1380-1419.

[3] J. Auvray, Identification de processus. Techniques de l'ingénieur, 1994, fiche r 306

[4] M. N. Özisik, Heat conduction, John Wiley and sons, 1980.

[5] W Parker, Flash method of determining thermal diffusivity, J Appl. Phys, 32, (9), 1961, 1679-1684

[6] J. Hladik, Métrologie des propriétés thermophysiques des matériaux, Masson, 1990.

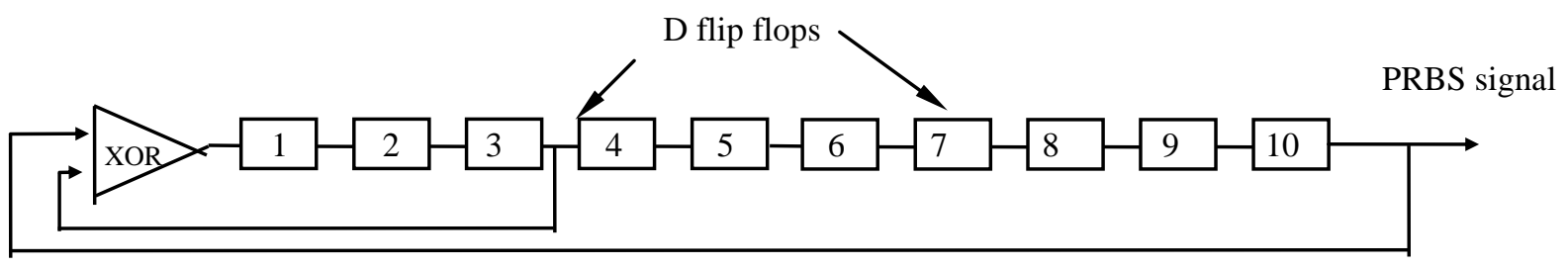

Figure 1: Principle of the pseudo - random binary sequence generation

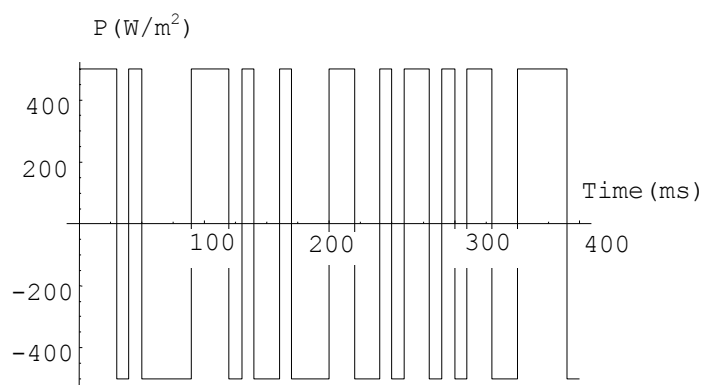

Figure 2: Example of an excitation sequence used 


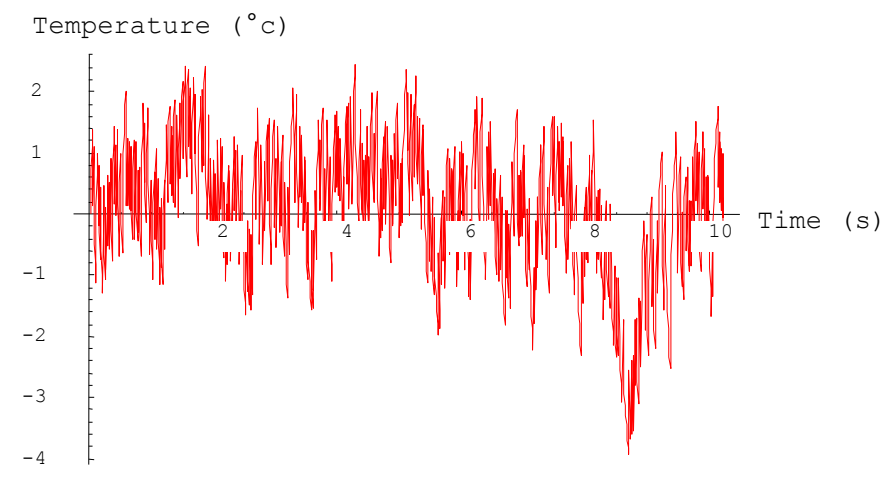

Figures 3: Thermal response of the duralumin sample studied

Pulse response $\left({ }^{\circ} \mathrm{C}\right)$

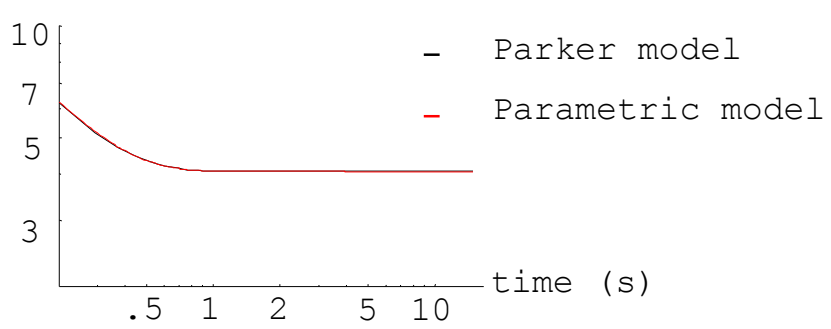

Figure 4: Comparison between real pulse response (obtained with the Parker model) and the estimated response (obtained with the parametric model) of a duralumin sample

Amplitude $\left({ }^{\circ} \mathrm{C}\right)$

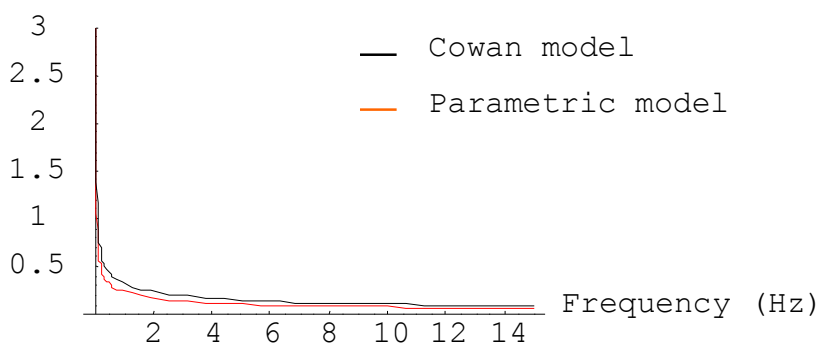

Phase $\left({ }^{\circ}\right)$

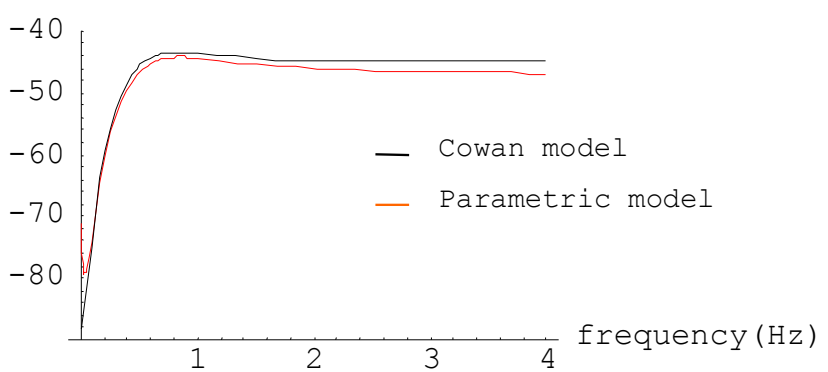

Figure 5: Comparison between real multifrequencies harmonic response (obtained with the Cowan model) and the estimated response (obtained with the parametric model) of a duralumin sample

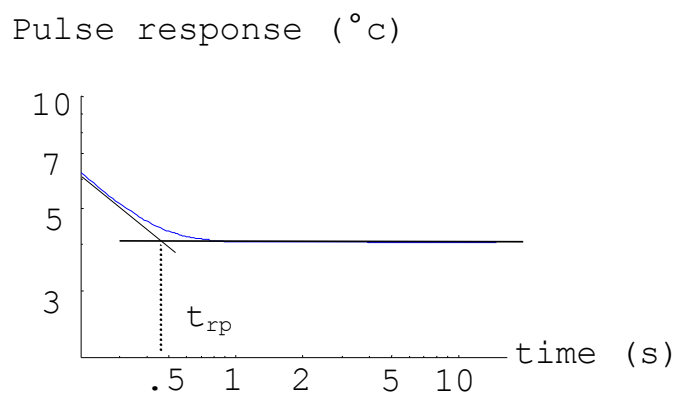

Figure 6: Determination, under lesser energetic constraint, of the thermal diffusivity of a duralumin sample, from its pulse response reconstructed by parametric analysis. 